Bond University

Research Repository

\title{
Process evaluation of a patient-centred, patient-directed, group-based education program for the management of type 2 diabetes mellitus
}

Odgers-Jewell, Kate; Isenring, Elisabeth; Thomas, Rae; Reidlinger, Dianne P.

\section{Published in:}

Nutrition and Dietetics

DOI:

10.1111/1747-0080.12327

Licence:

Other

Link to output in Bond University research repository.

Recommended citation(APA):

Odgers-Jewell, K., Isenring, E., Thomas, R., \& Reidlinger, D. P. (2017). Process evaluation of a patient-centred, patient-directed, group-based education program for the management of type 2 diabetes mellitus. Nutrition and Dietetics, 74(3), 243-252. https://doi.org/10.1111/1747-0080.12327

\section{General rights}

Copyright and moral rights for the publications made accessible in the public portal are retained by the authors and/or other copyright owners and it is a condition of accessing publications that users recognise and abide by the legal requirements associated with these rights.

For more information, or if you believe that this document breaches copyright, please contact the Bond University research repository coordinator. 


\section{Abstract}

2 Aim: This study developed and evaluated a patient-centred, patient-directed, group-

3 based education program for the management of type 2 diabetes mellitus.

4 Methods: Two frameworks, the MRC Framework for Developing and Evaluating

5 Complex Interventions and the RE-AIM framework were followed. Data to develop the

6 intervention were sourced from scoping of the literature and formative evaluation.

7 Program evaluation comprised analysis of primary recruitment of participants through

8 general practitioners, baseline and endpoint measures of anthropometry, four validated

9 questionnaires, contemporaneous facilitator notes and telephone interviews with

10 participants.

11 Results: A total of 16 participants enrolled in the intervention. Post intervention results were obtained from 13 participants with an estimated mean change from baseline in weight of $-0.72 \mathrm{~kg}$ (95\%CI -1.44 to -0.01$)$, BMI of $-0.25 \mathrm{~kg} / \mathrm{m}^{2}$ (95\%CI -0.49 to -0.01 ), and waist circumference of $-1.04 \mathrm{~cm}$ (95\%CI -4.52 to 2.44$)$. The group education program was acceptable to participants. The results suggest that recruitment through general practitioners is ineffective and alternative recruitment strategies are required.

Conclusions: This patient-centred, patient-directed, group-based intervention for the management of type 2 diabetes mellitus was both feasible and acceptable to patients. Health professionals should consider the combined use of the MRC and RE-AIM frameworks in the development of interventions to ensure a rigorous design process, and to enable the evaluation of all phases of the intervention, which will facilitate translation to other settings. Further research with a larger sample trialling additional recruitment strategies, evaluating further measures of effectiveness, and utilizing lengthier follow up periods is required. 
1

2 Diabetes is the fastest growing disease nationally and internationally, with one

3 Australian being diagnosed every eight minutes. ${ }^{1}$ Each year approximately 1 million

4 Australians are diagnosed with diabetes; 85\% with type 2 diabetes mellitus (T2DM). ${ }^{2}$

5 Patient education, the cornerstone of chronic disease self-management, is essential in

6 achieving improved outcomes and has been acknowledged as an integral and vital

7 component of successful T2DM care..$^{3,4,5,6}$ The main goal of diabetes patient education

8 is to promote and support positive self-management behaviours in order to optimize

9 metabolic control, improve quality of life (QOL), prevent acute and chronic complications, and reduce morbidity and mortality., 7

Group-based education for patients with T2DM has the potential to be a more cost effective and efficient intervention than individual education, due to the reduced time and funding required to educate numerous patients in one session. ${ }^{5,8}$ Group-based education allows time for the provision of more detailed information, decreases time demands on health workers, allows the easy incorporation of families and carers, and facilitates patient discussions and support from others in a similar situation. ${ }^{9,10}$ Research assessing the effectiveness of group-based education compared with usual care for the management of T2DM has found that the benefits in patient outcomes include significant improvements in glycaemic control, fasting blood glucose (FBG), diabetes knowledge, self-management skills, self-efficacy, and treatment satisfaction, as well as significant reductions in body weight, systolic blood pressure, and the need for diabetes medication. ${ }^{10,11}$ 
1 Despite the evidence supporting group-based education for the management of T2DM,

2 it is surprisingly difficult to define the ideal content and process by which effective

3 group-based education should be delivered. ${ }^{12}$ Group-based education programs can be

4 structured or unstructured, depending on the level of prescription in the content covered

5 and the delivery. Structured programs contain lesson plans with clearly defined content,

6 which can allow programs to be replicated by multiple group facilitators, however are

7 more likely than unstructured programs to utilize a didactic facilitation style, reducing

8 the time for patient interactions and discussion. ${ }^{13}$ Unstructured or patient-directed

9 programs utilize a non-didactic facilitation style and can allow participants to explore

10 their own agenda, interests and needs, rather than content that may not interest or assist

11 them in improving their self-management skills or knowledge. ${ }^{14}$

Within Australia, dietitians in particular overwhelmingly favour the provision of individual patient education services over group-based education. The utilization of group services for T2DM management provided by dietitians has continued to decrease in recent years whilst individual dietetic services have consistently increased. ${ }^{15}$ Previous research has proposed that service system issues, workforce capacity, awareness among practitioners and practitioner attitudes and preferences are the main factors impeding the utilization of group-based education by Australian dietitians. ${ }^{16}$ A recent study exploring group facilitators' perceptions and experiences of group-based chronic disease management programs found that interventions were being delivered with limited quality control and that facilitators had inadequate knowledge of the evidence base underpinning the programs they were facilitating. ${ }^{13}$ An additional surprising finding from this study was that the outcome measures being utilized by facilitators in practice 
1 were minimal, with many only collecting an overview of patient satisfaction through

2 surveys, which as a solitary measure, is inadequate in assessing patient outcomes or

3 improving the quality of future programs. ${ }^{13}$

5 The development of a group-based intervention informed by the literature and formative

6 research, followed by feasibility testing and a rigorous process evaluation may result in

7 an intervention that can be easily translated into practice by health professionals

8 interested in delivering group-based education programs and unsure where to start.

9 Additionally, the dissemination of findings from feasibility studies could contribute to

10 health practitioners' knowledge by furthering an understanding of the methodological and practical challenges of developing and implementing intervention studies in a 'realworld' setting, and may highlight outcome measures which are suitable for the evaluation of intervention effectiveness. ${ }^{17}$

The aim of this study was to develop and evaluate a patient-centred, patient-directed, group-based education program for the management of T2DM, using two process evaluation frameworks, the MRC Framework for Developing and Evaluating Complex Interventions, and the RE-AIM framework.

\section{Methods}

21 The development and process evaluation of the intervention using the two frameworks occurred over a number of phases (Figure 1). In brief this involved a formative evaluation including a scoping of the literature, interviews with facilitators and participants of existing chronic disease management group programs; recruitment and 
1 initial assessment of participants; baseline outcome measurements, the facilitation of the

2 intervention, follow up outcome measurements, and the completion of telephone

3 interviews with participants to assess the acceptability of the intervention.

$4 \quad$ [Insert figure 1 here]

5

6 The Medical Research Council (MRC) Framework for Developing and Evaluating

7 Complex Interventions (2008) was used to guide the intervention development and

8 evaluation. ${ }^{18}$ The framework incorporates four phases: development, feasibility and

9 piloting, evaluation and implementation, which aim to help researchers to recognise and

10 adopt appropriate measures for the design and evaluation of complex health behaviour

11 change interventions. ${ }^{18}$ The RE-AIM framework is an evaluation framework that

12 includes multiple process indicators to evaluate various aspects of an intervention:

13 reach, effectiveness, adoption, implementation and maintenance. ${ }^{19,} 20$ The RE-AIM

14 framework not only evaluates the effectiveness or strengths of an intervention, but also

15 the program's translatability, feasibility and limitations, which can potentially be

16 improved upon in future research. ${ }^{21}$ Combining both the MRC and RE-AIM frameworks

17 in the process evaluation of the intervention ensures a thorough and rigorous evaluation

18 of all aspects of the program including development, and enables the identification of

19 strengths and limitations.

21 To develop the intervention data were collected from three sources: a preliminary

22 literature review and scoping of group-based interventions for T2DM management, a

23 formative evaluation based on interviews with facilitators of a range of existing chronic

24 disease management (CDM) group education programs and their participants, and a 
1 review of the Medicare group services information pack, which is evidence-based,

2 available to Australian health-professionals, and is likely to influence the development

3 of group-based education programs in practice. ${ }^{22}$ Triangulation was achieved by

4 comparing the attributes of effective group-based education interventions with the

5 results obtained from the group facilitator and group participant interviews, and the

6 information provided in the Medicare group services information pack. ${ }^{22}$ Triangulation

7 is commonly utilized in health service research as an evaluation method as it enables the

8 integration of methods and approaches to conduct better evaluation studies. ${ }^{23}$

The intervention design, resulting from the systematic development process, was a patient-centred, patient-directed, group-based education program. The program content employed a non-didactic approach, group discussions were encouraged, and the content covered in the group education sessions was decided by group participants in the first session. The intervention is described using the Template for Intervention Description and Replication (TIDieR) checklist and guide (Supporting Information: Appendix I). ${ }^{24}$

A recruitment target of 90 participants for the single-arm feasibility study was set. The sample size, although not necessary for a feasibility study, was determined from a practice perspective. The sample size of 90 participants was originally calculated for a two-armed study, in which each intervention group would be composed of 45 participants, allowing for at least 20\% attrition, resulting in three groups of 12 participants (per intervention group). General Practitioner (GP) referrals were chosen as the primary recruitment strategy for the feasibility study, based on literature suggesting they are the 'gatekeepers' of primary care, and the initial point of contact for patients 
1 who require primary or non-emergency health care. ${ }^{25}$ Invitation letters were mailed to

2 all medical centres $(\mathrm{n}=132)$ within a $50 \mathrm{~km}$ radius of the intervention site and each

3 medical centre was telephoned to follow up within two weeks of postage.

4

5 Participants were included if they self-reported a diagnosis of T2DM or were referred 6 by their GP as a diagnosed T2DM patient, were 18 years of age or over, had adequate 7 cognitive ability, and had a sufficient understanding of English. Ethical approval was

8 obtained from the Bond University Human Research Ethics Committee (protocol 9 number RO1815), and written informed consent was obtained from each participant 10 prior to the commencement of the intervention, which was provided free of charge.

12 An Accredited Practising Dietitian (KOJ) conducted all of the initial consultations and intervention sessions. Participants attended an initial individual consultation to assess whether they met the inclusion criteria, and to obtain demographic and baseline data. Group-based education sessions were conducted at a local community centre to ensure easy access for group participants. The participants were allocated to one of two groups; both groups were facilitated using the same approach. Group allocation depended on participant availability and to ensure participant numbers were fewer than 12 per group to align with the Medicare CDM group service item guidelines. Groups were facilitated on a weekday morning for two hours for a six-week period.

The group intervention was evaluated using process and participant measures including questionnaires and anthropometric data to assess the feasibility of the intervention, and semi-structured interviews with group participants to assess the acceptability of the 
1 intervention. Additionally, the group facilitator (KOJ), kept a researcher journal

2 throughout the intervention to record reflections and logistics such as participant

3 attendance, suitability of the venue, and peer interactions.

4

5 Baseline (2-3 weeks prior to commencing the intervention) and endpoint data (taken

6 during the final group session of the program) included weight, waist circumference and

7 height measurements, and four validated questionnaires assessing nutrition

8 knowledge $^{26}$, diabetes self-efficacy ${ }^{27}$, diabetes knowledge ${ }^{28}$ and diabetes-related QOL. ${ }^{29}$

9 Only the first two sections (related to dietary recommendations and nutrient sources)

10 from the nutrition knowledge questionnaire ${ }^{26}$ were administered, due to the relevance

11 and length of the questionnaire.

13 Data were assessed for normality and analysed, where appropriate, using the statistical 14 package SPSS (Statistical package for the social sciences, version 23.0). Prior to 15 analysis, each of the data sets was assessed for normality. Normally distributed data was analysed using paired sample t-tests to assess differences in the baseline and endpoint measures of the group participants for the five normally distributed measures. Wilcoxon-signed rank tests were performed on two measures that were not normally 19 distributed.

21 The adoption, implementation and acceptability of the intervention were measured by the number of face-to-face sessions attended and by individual telephone interviews conducted by an independent research assistant following the completion of the groupbased intervention. The interview questions were developed from earlier research 
1 (Odgers-Jewell, et al. 2016, unpublished data). ${ }^{13}$ The interviews were audio-recorded,

2 transcribed, checked, anonymised and corrected against the audio files by the first

3 author (KOJ). Content was extracted from the interview transcripts by the first author

4 and confirmed with the senior author (DPR) in order to answer the pre-defined set of

5 questions, which explored the acceptability of the intervention. Responses to the

6 demographic questions were categorized and enumerated.

7

\section{Results}

9 Three sources were used to develop the intervention. The literature review indicated that

10 patient-centred group education with the following attributes were favoured: patient

11 involvement in the design, planning, goal setting and decision making process, regular

12 reinforcement after education, individualised content, and non-didactic facilitation by an

13 individual or multidisciplinary team or peer leaders. ${ }^{10,} 11$ These were combined with

14 information provided to allied health professionals in the Medicare group services

15 information pack including the need for programs to be: patient-centred, facilitated by a

16 multidisciplinary team, developed according to a plan with achievable and measurable

17 goals and objectives, to incorporate group rules, and to allocate time for patients to

18 discuss their experiences. ${ }^{22}$ Finally, formative interviews with group facilitators and

19 participants from existing CDM group education programs indicated: a preference for a

20 strong focus on group interactions by providing patients with a non-didactic, interactive,

21 discussion-based program; the importance of group rules set at the commencement of the group-based education sessions; and goal-oriented and patient-centred content. 
1 After triangulating these data, the elements used in the development of the final

2 intervention included a non-didactic, patient-centred approach, the incorporation of

3 group rules, and adequate time for group discussions. There was a lack of consensus on

4 the materials or educational content ideally provided to patients in a group-based

5 education setting, suggesting that more emphasis should be placed on encouraging

6 group interactions, rather than a sole focus on the content of sessions. There was

7 divergence in the appropriate length and number of sessions, however two-hour

8 sessions were chosen as the literature review and formative interviews indicated that

9 this was an appropriate amount of time to allow group participants to have in depth

10 discussions. Additionally, it was decided that the sessions would run for six weeks -

11 again to align with the findings of the literature review and formative interviews, and to

12 ensure that the time commitment from patients was not unreasonable.

14 Recruitment targets through GPs were not met: only two out of 132 (1.5\%) medical 15 centres responded to multiple requests to display recruitment flyers in waiting rooms or 16 consultation rooms. Due to this low response rate, alternative strategies were used 17 including advertisements and stories in two local newspapers, recruitment flyers in six 18 local pharmacies, and an advertisement on the University website. Group participants 19 were all recruited through feature stories in a free local newspaper.

21 An accurate estimation of the reach of the recruitment strategy was not possible, 22 however it is estimated that the number of persons diagnosed with T2DM within the $2350 \mathrm{~km}$ recruitment radius would be approximately 950 persons. ${ }^{30}$ Thirty-three 24 (approximately 3.5\% of the estimated area population with T2DM) potential 
1 participants made initial contact with the researcher of which a total of 16 participants

2 enrolled in the study. Three did not complete the intervention (Figure 2).

$3 \quad$ [Insert figure 2 here]

4

5 Demographics of the 13 group participants who attended the program and completed

6 the telephone interviews are presented in Table 1 . The intervention participants were

7 predominantly Australian, however some participants were born in Europe (United

8 Kingdom, Croatia, France, Poland and Germany).

$9 \quad$ [Insert Table 1 here]

11 The results of the four questionnaires and anthropometric measures are shown in Table 2. The results suggest reductions in body weight, BMI, waist circumference, and

13 increased diabetes knowledge, nutrition knowledge, diabetes self-efficacy, and diabetes-

14 related QOL. However, despite two of these outcomes reaching statistical significance, 15 the small sample size of the study was not sufficiently powered to reliably detect 16 significant statistical differences.

17 [Insert Table 2 here]

19 The key results of the process evaluation of the intervention study are summarized in 20 Table 3.

21 [Insert Table 3 here]

23 Telephone interviews were used to explore the acceptability of the program, 24 participants' preferences for group program structure and facilitation, and their 
1 perceptions of the effect of group interactions. The group-based intervention was

2 acceptable, with all group participants stating that they would recommend the program

3 to friends or family as they found it informative, indicated they enjoyed speaking with

4 other people who had been diagnosed with T2DM, and found the information provided

5 interesting. Participants noted aspects they liked most were: group interactions, the

6 facilitators' relaxed attitude, and the length of the group program. A few participants

7 stated a preference for sessions where they perceived that discussions remained on the

8 agreed topic. Participants frequently reported that other group members helped their

9 learning through peer identification and from others' experiences. Participants from

10 both groups exchanged contact details at the completion of the intervention with the

11 intention to maintain contact beyond the program.

13 Discussion

14 This study reports on the process evaluation of a single-arm patient-centred, patient-

15 directed, group-based education program, and this paper has described its development,

16 feasibility testing and evaluation. Two frameworks were used to capture each phase of

17 the development and evaluation. The triangulation of data from three sources resulted in

18 the development of a non-didactic, patient-centred intervention, which was delivered to

19 participants weekly for a six-week period. The results of the evaluation suggest that the

20 intervention was feasible, and acceptable to the target group. However, the recruitment

21 strategy was inadequate and resulted in an insufficient reach of the target population.

22 As such, the maintenance phase of the RE-AIM framework, or the equivalent

23 implementation phase of the MRC Framework for Developing and Evaluating Complex 
1 Interventions, could not be explored.

2

3 Group education research has established the ineffectiveness of didactic education

4 techniques when compared to non-didactic patient education. ${ }^{11,31}$ Evidence supports the

5 use of a patient-centred approach, care that is respectful of, and responsive to,

6 individual patient preferences, needs and values, and has shown that engaging patients

7 in their health care decisions can enhance their adherence to therapy. ${ }^{32}$ Within T2DM,

8 patient-centred interventions have been effective in improving patient knowledge, blood

9 glucose levels, weight, and medication usage, and have been shown to improve self-

10 management behaviours. ${ }^{17}$ A patient-directed approach, in which the content of the group-education program is decided by the patients, reflects patients' own needs and questions, and includes discussions initiated by patients, has been successfully utilized by various group-based education studies for the management of T2DM. ${ }^{33}, 34$ Allowing patients to direct their own learning through negotiated topics proposed by group members may support self-management.

17 A key finding and limitation of this feasibility study was the ineffectiveness of recruiting people with T2DM through GPs. The overall poor recruitment rate may have been due to the use of GPs as a primary strategy and the generally low uptake of group education programs by T2DM patients. Despite their principal role in the management of T2DM patients in the primary health care setting, engaging GPs and recruiting participants through GPs was difficult. Barriers to recruitment via GPs have previously been suggested as time and workload pressures ${ }^{35,36}$, negative attitudes towards research, concerns about researchers' motives, a lack of interest in the topic of research, and a 
1 lack of recognition. ${ }^{37}$ Monetary and nonmonetary incentives, endorsement by relevant

2 authorities, and multiple reminder contacts with clinicians may have boosted research

3 response rates. ${ }^{38}$ In addition, clinicians may have felt overwhelmed with requests for

4 research participation, desired a greater involvement in the study, or been concerned

5 about the potential lack of effectiveness of a new trial that would not be an ongoing

6 addition to the health care system. ${ }^{39}$

7

8 The generally poor uptake of group-based programs for the management of T2DM may

9 have also contributed to the reduced recruitment. ${ }^{30,40-42}$ A recent study found that the

10 three main reasons for non-attendance of group-programs as reported by T2DM patients

11 were the lack of information or perceived benefit of the programs, unmet personal preferences such as poor timing or accessibility of group locations, and the shame and stigma of diabetes. ${ }^{43}$ Practitioners should consider how health professionals in primary care communicate with their T2DM patients in regards to group-education programs, the optimal timing and location of group programs, and focus on recruitment methods that minimise any health-related stigma around T2DM. ${ }^{43}$

The evaluation found modest improvements in body weight, BMI and waist circumference as well as the quality of life domains, nutrition knowledge, diabetes knowledge and self-efficacy measures. Despite the improvements in these measures, the results should not be overstated due to the small sample size, short follow-up period and natural fluctuations in weight, BMI and waist circumference which may have occurred over the same time period. Feasibility study results should in general be interpreted cautiously, as effects may be smaller or more variable when a full-scale study is 
1 conducted. ${ }^{18}$ The effectiveness of feasibility studies should primarily be measured using

2 descriptive statistics, qualitative analysis and basic process evaluation data such as

3 administrative data. ${ }^{44}$

4

5 The participant evaluation component of this feasibility study, through interviews with

6 each participant, provided insightful and valuable data from which various conclusions

7 can be drawn. These included satisfaction with the intervention, willingness of patients

8 to recommend the intervention, and the positive evaluations of group interactions.

9 Patient satisfaction has been shown to be clinically relevant, with satisfied patients

10 being more likely to comply with treatment and to self-manage their condition. ${ }^{45}$ The

11 majority of group participants found other group members added to their learning,

12 generally through peer identification and learning from others' experiences. Providing

13 social support to patients with T2DM has been shown to extensively affect patient

14 behaviour. ${ }^{46}$ In particular, group interactions and peer identification may promote self-

15 efficacy, self-esteem, self-perception, awareness, and positive attitudes towards T2DM

16 and reduce disease-related anxiety. ${ }^{46,} 48$ The group interactions and discussions

17 encouraged in this study are likely to have had a positive impact on the acceptability

18 and effectiveness of the intervention.

20 Conducting a feasibility study, which trials components of a randomized controlled trial

21 (RCT), as opposed to a pilot study which trials the operation of all aspects of the developed RCT, allows researchers to assess the design, methodology and feasibility of

23 a larger pilot study, and to identify and prepare for the challenges of evaluating an

24 intervention. ${ }^{17,} 44$ Intervention studies are commonly plagued with problems of 
1 acceptability, compliance, delivery of the intervention, recruitment and retention, and

2 smaller than expected effect sizes, which could have been predicted, and potentially

3 avoided, through feasibility testing and piloting. ${ }^{17}$ Feasibility testing an intervention

4 prior to completing a pilot study additionally allows researchers to assess the

5 acceptability of an intervention and enhances the scientific rigour of the larger study. ${ }^{17}$

6

7 There were a number of strengths of the study. The utilization of two complementary

8 development and process evaluation frameworks enabled a comprehensive evaluation of

9 all aspects of the program, and may provide a useful guide for the development of

10 interventions in future. The developed intervention reflects facilitator-patient contact time that is suitable for Australian health professionals planning to facilitate groupbased education programs through the Medicare CDM group service rebates. The implementation of the intervention in a real-world setting enabled the researchers to explore the feasibility of the program in the context in which diabetes is usually managed. The inclusion of interviews to assess the acceptability of the intervention from the perspective of group participants, and the inclusion of participants from a range of backgrounds and with a range of years since diagnosis, ensured participant evaluation was robust.

There were also several limitations. Recruitment utilizing GPs as a primary recruitment strategy was unsuccessful, however this resulted in key learnings, which may be applied when translating the program to practice. Recruitment to future interventions may be improved through the additional use of specialist clinics, such as diabetes outpatient clinics, which utilize electronic health records enabling the identification and 
1 monitoring of participants ${ }^{30}$, involving participants in trial design ${ }^{49}$, using shorter and

2 more informative recruitment flyers $^{50}$, and providing monetary incentives to

3 participants. ${ }^{51}$ The potential for sampling bias cannot be ruled out - the sample

4 characteristics of the group participants were dissimilar to the characteristics of

5 participants in the AusDiab study. ${ }^{52}$

6

7 This process evaluation indicated that a patient-centred, patient-directed, group-based

8 intervention for the management of T2DM was both feasible and acceptable to patients.

9 Additionally, a number of factors were identified as requiring refinement prior to the

10 facilitation of a pilot study, particularly in regards to recruitment issues. Health

11 professionals should consider the use of the RE-AIM and MRC frameworks in the

12 development of group-based interventions to ensure a thorough and complete design,

13 and evaluation of all phases of the intervention. Furthermore, describing an intervention

14 using the TIDieR checklist and guide can improve the completeness of intervention

15 reporting and enable replicability. ${ }^{24}$ Further research trialling additional recruitment

16 strategies, evaluating further measures of effectiveness, and utilizing lengthier follow up

17 periods is required. 


\section{References:}

2 1. Dietitians Association of Australia (DAA). Evidence Based Practice Guidelines

3 for the Nutritional Management of Type 2 Diabetes Mellitus for Adults. DAA, Canberra 42006.

5 2. Australian Institute of Health and Welfare (AIHW). Australia's Health 2014.

6 Australia's health series no. 14. Cat. no. AUS 178. Canberra: AIHW, 2014.

$7 \quad$ 3. Adolfsson ET, Starrin B, Smide B, Wikblad K. Type 2 diabetic patients'

8 experiences of two different educational approaches-A qualitative study. Int $J$ Nurs

$9 \quad$ Stud. 2008;45(7):986-94.

10 4. Ellis SE, Speroff T, Dittus RS, Brown A, Pichert JW, Elasy TA. Diabetes

11 patient education: a meta-analysis and meta-regression. Patient Educ Couns.

$12 \quad$ 2004;52(1):97-105.

13 5. Mensing CR, Norris SL. Group education in diabetes: effectiveness and 14 implementation. Diabetes Spectr. 2003;16(2):96-103.

15 6. Diabetes Australia. National Evidence Based Guideline for Patient Education in 16 Type 2 Diabetes. Sydney: Diabetes Australia Guideline Development Consortium 17 (DAGD), 2009.

7. $\quad$ Norris SL, Lau J, Smith SJ, Schmid CH, Engelgau MM. Self-Management

19 education for adults with type 2 Diabetes A meta-analysis of the effect on glycemic

20 control. Diabetes Care. 2002;25(7):1159-71.

21 8. Heller SR, Clarke P, Daly H, Davis I, McCulloch DK, Allison SP, Tattersall RB.

22 Group education for obese patients with type 2 diabetes: greater success at less cost.

23 Diabet Med. 1988 Sep 1;5(6):552-6. 
19 WHO. Peer support programmes in diabetes. Geneva: World Health

2 Organization, 2007.

3 10. Steinsbekk A, Rygg LØ, Lisulo M, Rise MB, Fretheim A. Group based diabetes

4 self-management education compared to routine treatment for people with type 2

5 diabetes mellitus. A systematic review with meta-analysis. BMC Health Serv Res.

6 2012;12(1):1.

7 11. Deakin T, McShane CE, Cade JE, Williams R. Group based training for self-

8 management strategies in people with type 2 diabetes mellitus. Cochrane Database Syst

$9 \quad$ Rev. 2005;2.

10 12. Skinner T, Carey M, Cradock S, et al. 'Educator talk'and patient change: some

11 insights from the DESMOND (Diabetes Education and Self Management for Ongoing

12 and Newly Diagnosed) randomized controlled trial. Diabet Med. 2008;25(9):1117-20.

13 13. Odgers-Jewell K, Hughes R, Isenring E, Desbrow B, Leveritt M. Group

14 facilitators' perceptions of the attributes that contribute to the effectiveness of

15 group-based chronic disease self-management education programs. Nutrition \&

16 Dietetics. 2015;72(4):347-55.

17 14. Hornsten A, Stenlund H, Lundman B, Sandstrom H. Improvements in HbA1c

18 remain after 5 years- a follow up of an educational intervention focusing on patients'

19 personal understandings of type 2 diabetes. Diabetes Res Clin Pract. 2008;81:50-5.

20 15. Cant R, Ball L. Decade of Medicare: The contribution of private practice

21 dietitians to chronic disease management and diabetes group services. Nutrition \&

22 Dietetics. 2015;72(3):284-90. 
1 16. Cant, R., Foster, M. Update on Medicare-funded allied health chronic disease

2 management consultation in dietetics and the five most referred professions in 2010.

$3 \quad$ Nutrition \& Dietetics, 2013. 70: p. 42-48.

4 17. Feeley N, Cossette S, Côté J, et al. The importance of piloting an RCT

5 intervention. CJNR. 2009;41(2):84-99.

6 18. Craig P, Dieppe P, Macintyre S, Michie S, Nazareth I, Petticrew M. Developing

7 and evaluating complex interventions: the new Medical Research Council guidance.

$8 \quad$ BMJ. 2008;337:a1655.

9 19. Glasgow RE, Vogt TM, Boles SM. Evaluating the public health impact of health 10 promotion interventions: the RE-AIM framework. Am J Public Health.

11 1999;89(9):1322-7.

12 20. Dzewaltowski DA, Glasgow RE, Klesges LM, Estabrooks PA, Brock E. RE-

13 AIM: evidence-based standards and a Web resource to improve translation of research

14 into practice. Ann Behav Med. 2004;28(2):75-80.

15 21. Lakerveld J, Bot S, Chinapaw M, van Tulder M, Kingo L, Nijpels G. Process

16 evaluation of a lifestyle intervention to prevent diabetes and cardiovascular diseases in

17 primary care. Health Promot Pract. 2012:1524839912437366.

22. Medicare Australia. Type 2 Diabetes: Medicare group services information pack.

19 Canberra: Dietitians Association of Australia, 2007 (Available from

20 http://daa.asn.au/for-health-professionals/medicare/:, accessed 28 May 2014).

21 23. Ammenwerth E, Iller C, Mansmann U. Can evaluation studies benefit from triangulation? A case study. Int J Med Inf. 2003;70(2):237-48. 
1 24. Hoffmann TC, Glasziou PP, Boutron I, et al. Better reporting of interventions:

2 template for intervention description and replication (TIDieR) checklist and guide.

$3 \quad$ BMJ. 2014;348:g1687.

4 25. Ball L, Hughes R, Leveritt M. Health professionals' views of the effectiveness of

5 nutrition care in general practice setting. Nutrition \& Dietetics. 2013;70(1):35-41.

6 26. Hendrie GA, Coveney J, Cox D. Exploring nutrition knowledge and the

7 demographic variation in knowledge levels in an Australian community sample. Public

$8 \quad$ Health Nutr. 2008;11(12):1365-71.

9 27. McDowell J, Courtney M, Edwards H, Shortridge-Baggett L. Validation of the 10 Australian/English version of the Diabetes Management Self-Efficacy Scale. Int J Nurs Prac. 2005;11(4):177-84.

28. Fitzgerald JT, Funnell MM, Hess GE, et al. The reliability and validity of a brief diabetes knowledge test. Diabetes Care. 1998;21(5):706-10.

29. Boyer JG, Earp JAL. The development of an instrument for assessing the quality of life of people with diabetes: Diabetes-39. Med Care. 1997;35(5):440-53.

30. van Staa T-P, Goldacre B, Gulliford M, et al. Pragmatic randomised trials using

17 routine electronic health records: putting them to the test. BMJ. 2012;344:e55.

31. Deakin T, Cade J, Williams R, Greenwood D. Structured patient education: the Diabetes X-PERT Programme makes a difference. Diabet Med. 2006;23(9):944-54. 32. Inzucchi SE, Bergenstal RM, Buse JB, et al. Management of hyperglycemia in type 2 diabetes: a patient-centered approach position statement of the American Diabetes Association (ADA) and the European Association for the Study of Diabetes (EASD). Diabetes Care. 2012;35(6):1364-79. 
1 33. Hörnsten Å, Lundman B, Stenlund H, Sandström H. Metabolic improvement

2 after intervention focusing on personal understanding in type 2 diabetes. Diabetes Res

$3 \quad$ Clin Pr. 2005;68(1):65-74.

4 34. Sarkadi A, Rosenqvist U. Experience-based group education in Type 2 diabetes:

5 a randomised controlled trial. Patient Educ Couns. 2004;53(3):291-8.

6 35. Kaner E, Haighton CA, McAvoy BR. 'So much post, so busy with practice--so,

7 no time!': a telephone survey of general practitioners' reasons for not participating in

8 postal questionnaire surveys. Br J Gen Pract. 1998;48(428):1067-9.

9 36. Stocks N, Gunnell D. What are the characteristics of general practitioners who

10 routinely do not return postal questionnaires: a cross sectional study. Journal Epidemiol

11 Community Health. 2000;54(12):940-1.

12 37. Hummers-Pradier E, Scheidt-Nave C, Martin H, Heinemann S, Kochen MM,

13 Himmel W. Simply no time? Barriers to GPs' participation in primary health care

14 research. Fam Pract. 2008;25(2):105-12.

15 38. VanGeest JB, Johnson TP, Welch VL. Methodologies for improving response

16 rates in surveys of physicians a systematic review. Eval Health Prof. 2007;30(4):303-

$17 \quad 21$.

18 39. Fletcher B, Gheorghe A, Moore D, Wilson S, Damery S. Improving the

19 recruitment activity of clinicians in randomised controlled trials: a systematic review.

20 BMJ Open. 2012;2(1):e000496.

21 40. Sturt JA, Whitlock S, Fox C, et al. Effects of the Diabetes Manual 1: 1 structured education in primary care. Diabet Med. 2008;25(6):722-31. 
1 41. Wadher, K ed. Service review of DESMOND non-attenders 2010: How

2 attendance of structured group education for type 2 diabetes can be improved.

3 Birmingham, UK: Primary Care Diabetes Society, 2010.

4 42. Glasgow RE, Toobert DJ, Hampson SE. Participation in Outpatient Diabetes

5 Education Programs: How Many-Patients Take Part and How Representative Are They?

$6 \quad$ Diabetes Educ. 1991;17(5):376-80.

7 43. Winkley K, Evwierhoma C, Amiel S, Lempp H, Ismail K, Forbes A. Patient

8 explanations for non-attendance at structured diabetes education sessions for newly

9 diagnosed Type 2 diabetes: a qualitative study. Diabet Med. 2015;32(1):120-8.

10 44. Tickle-Degnen L. Nuts and bolts of conducting feasibility studies. Am J Occup

11 Ther. 2013;67(2):171-6.

12 45. Asadi-Lari M, Tamburini M, Gray D. Patients' needs, satisfaction, and health related quality of life: towards a comprehensive model. Health Qual Life Outcomes. 2004;2(1):1.

46. Cooper H, Booth K, Fear S, Gill G. Chronic disease patient education: lessons from meta-analyses. Patient Educ Couns. 2001;44(2):107-17.

47. Raballo M, Trevisan M, Trinetta AF, et al. A Study of Patients' Perceptions of

Diabetes Care Delivery and Diabetes Propositional analysis in people with type 1 and 2 diabetes managed by group or usual care. Diabetes Care. 2012;35(2):242-7.

48. Trento M, Passera P, Borgo E, et al. A 5-year randomized controlled study of learning, problem solving ability, and quality of life modifications in people with type 2 diabetes managed by group care. Diabetes Care. 2004;27(3):670-5. longitudinal study. Brit J Psychiat. 2013;203(5):381-6. 
$150 . \quad$ Edwards SJ, Lilford RJ, Thornton J, Hewison J. Informed consent for clinical

2 trials: in search of the "best” method. Soc Sci Med. 1998;47(11):1825-40.

3 51. Watson JM, Torgerson DJ. Increasing recruitment to randomised trials: a review

4 of randomised controlled trials. BMC Med Res Methodol. 2006;6(1):34.

5 52. Barr ELM, Magliano PJ, Zimmet PZ, et al. AusDiab 2005: The Australian

6 Diabetes, Obesity and Lifestyle Study. Melbourne: International Diabetes Institute, 72005. 
Table 1: Group participant sample attributes summary (n=13)

\begin{tabular}{ll}
\hline Attribute & N \\
\hline Gender: & \\
Male & 7 \\
Female & 6
\end{tabular}

Age:

$\begin{array}{ll}55-64 \text { yrs } & 3 \\ 65-74 \text { yrs } & 5 \\ \geq 75 \text { yrs } & 5\end{array}$

Marital Status:

$\begin{array}{ll}\text { Married } & 8 \\ \text { Divorced } & 2 \\ \text { Separated } & 1 \\ \text { Widowed } & 2\end{array}$

Education:

$\begin{array}{ll}\text { Primary } & 1 \\ \text { Secondary } & 6 \\ \text { Tertiary } & 6\end{array}$

Employment:

$\begin{array}{ll}\text { Casual } & 1 \\ \text { Self-employed } & 1 \\ \text { Retired } & 11\end{array}$

Years since diagnosis:

$\begin{array}{ll}<1 \mathrm{yr} & 2 \\ 1-3 \mathrm{yrs} & 2 \\ 4-6 \mathrm{yrs} & 4 \\ 7-9 \mathrm{yrs} & 2 \\ \geq 10 \mathrm{yrs} & 3\end{array}$

Previous group attendance: 11 
Table 2: Change in anthropometry and questionnaire outcomes $(n=13)$

\begin{tabular}{lcc}
\hline Paired t-tests & $\begin{array}{c}\text { Pre-intervention } \\
\text { Mean (SD) }\end{array}$ & $\begin{array}{c}\text { Post-intervention } \\
\text { Mean (SD) }\end{array}$ \\
\hline Body weight (kg) & $87.1(14.88)$ & $86.4(14.52)^{*}$ \\
BMI (kg/m²) & $30.5(5.3)$ & $30.3(5.22)^{*}$ \\
Waist circumference (cm) & $108.7(16.29)$ & $107.7(17.44)$ \\
Diabetes Knowledge $^{(a)}$ & $13.38(4.13)$ & $13.92(4.19)$ \\
Diabetes-related QOL $^{(a)}$ & $121.5(47.42)$ & $112.08(46.63)$ \\
\hline Wilcoxon-signed rank tests & Pre-intervention & Post-intervention \\
& Mean (SD) & Mean rank (SD) \\
\hline Nutrition Knowledge $^{(a)}$ & $44.77(11.56)$ & $47.54(7.83)$ \\
Diabetes Self-Efficacy $^{(\text {a) }}$ & $60.6(17.96)$ & $67.15(12.88)$ \\
\end{tabular}

(a) Improved scores post-intervention, ns

* Indicates post intervention measures were assessed as significant $(\mathrm{P} \leq 0.05)$ 


\section{Table 3: Summary table of evaluation results}

\begin{tabular}{|c|c|}
\hline $\begin{array}{l}\text { MRC framework for } \\
\text { complex interventions }^{16}\end{array}$ & Key Findings \\
\hline \multirow[t]{6}{*}{ Development phase } & Literature scoping \\
\hline & $\begin{array}{l}\text { Two systematic reviews }{ }^{9,10} \text { : recommendations were } 5 \text { to } 16 \text { participants per group; } 8 \text { to } 52 \text { hours of facilitator } \\
\text { patient contact time over } 6 \text { to } 12 \text { sessions }\end{array}$ \\
\hline & Medicare group services information pack ${ }^{20}$ : recommendations were 2 to 12 participants per group, minimum \\
\hline & $8 \mathrm{x}$ one hour sessions ( 8 hours of facilitator-patient contact time), individual assessment prior to commencem \\
\hline & Formative evaluation \\
\hline & $\begin{array}{l}\text { Facilitator and participant interviews: recommendations were } 5 \text { to } 25 \text { participants per group; } 10 \text { to } 24 \text { hours o } \\
\text { contact time over } 4 \text { sessions }\end{array}$ \\
\hline
\end{tabular}

\section{Feasibility and piloting}

\section{Recruitment of participants}

Reach

33 potential participants made initial contact with the researcher; a total of 16 participants enrolled (3 dropouts) in the study; 13 completed the study (14.4\% of the initial target)

\section{Initial assessment}

100\% met the inclusion criteria, were suitable to participate and provided demographic data

\section{Evaluation}

\section{Evaluation}

Implementation $^{(a)}$
Baseline measures- anthropometry

Mean body weight $( \pm \mathrm{SD})(\mathrm{kg})$ : 87.1 (14.88); Mean BMI $( \pm \mathrm{SD})\left(\mathrm{kg} / \mathrm{m}^{2}\right)$ : 30.5 (5.3); Mean waist circumference $( \pm \mathrm{SD})(\mathrm{cm}): 108.7(16.29)$

\section{Delivery of intervention}

Participants from both groups attended 4 to 6 (67-100\% attendance) sessions; those who missed sessions were unable to attend due to other medical appointments, illness or travel plans.

\section{Follow up measures- anthropometry}

Mean body weight $( \pm \mathrm{SD})(\mathrm{kg})$ : 86.4 (14.52); Mean BMI $( \pm \mathrm{SD})\left(\mathrm{kg} / \mathrm{m}^{2}\right)$ : 30.3 (5.23); Mean waist circumference ( \pm SD) (cm): $107.7(17.44)$

Interviews

Program structure:
RE-AIM process evaluation

\section{framework 17,18}

Implementation

Effectiveness

Adoption 
Aspects liked least: Discussions can go off topic

Recommended changes: Program could have gone for longer

Ideal program length: 6 weeks, for 2 hours per week (as delivered)

Group interactions:

Helped/ hindered learning: Helped; Peer identification and learning from others' experiences

Role of group facilitator: Facilitating the group; Explaining points

Patient satisfaction:

Recommend program: Yes

(a) The implementation phase of the MRC framework refers to aspects of maintenance more aligned with the adoption and maintenance phases of the RE-AIM framework 


\section{Appendix I: Intervention study design using the TIDieR Checklist and Guide}

\section{Why}

3

What: Materials

Procedures

\section{Who provided}

The Bond Diabetes Intervention (ANZCTR registration:12614000572662)

Rationale or theory: Patient-centred care, non-didactic approach, patient-directed intervention

Goal: To evaluate the feasibility and acceptability of the Bond Diabetes Intervention using two process evaluation frameworks (MRC Framework for Developing and Evaluating Complex Interventions and RE-AIM)

Participants were provided with freely available handouts from the Diabetes Queensland website ${ }^{1}$ and the Australian Government Department of Health Eat for Health website²; and simple recipes were given to the participants of group 2 only at the request of group members

Participants were phoned by the group facilitator a week before the commencement of the program to remind them of the commencement date and location.

The first session commenced with an introduction by the facilitator, followed by introductions by each of the participants who also explained when they were diagnosed with T2DM. A brainstorming process was used whereby suggested topics were transcribed onto a whiteboard, and then assigned to the sessions to guide content. The topics chosen varied slightly between groups:

Group $1(n=7)$ selected understanding diabetes, medications, diet and glycaemic index, hunger pains, margarines and spreads, controlling and checking blood glucose levels (BGLs), BGL diaries, and feeling overwhelmed or stressed;

Group $2(\mathrm{n}=6)$ selected glycaemic index, gluten free food, sugar cravings, reading food labels, BGL testing, exercise, HbA1c levels, medications, simple recipes, and what to eat.

Group rules, informed by previously published 'responsibilities of the group', were established at the first session and discussed to ensure that all participants were aware of expectations. ${ }^{2}$ These rules included: 1 . Come to every session, 2. Ask anything you want, 3. Maintain confidentiality, and 4. Give new activities at least a 2-week trial. ${ }^{3}$

A short break was taken in the middle of each session for morning tea, which was provided, in order to allow the participants to become better acquainted in a relaxed environment.

The final session included a summary of the topics and farewell.

Provider: Accredited Practising Dietitian (KOJ); Training: Informal group education training (online) and formal training during University degree (Master of Nutrition and Dietetics, Griffith University) and professional placements across Individual Case Management.

Face-to-face, group-based, non-didactic delivery using a discussion based, patient-centred and patient directed approach. 


\section{$9 \quad$ Tailoring}

10 Modifications

11 How well: Planned

12 Actual
Personalization: The intervention was personalized in that participants received the first session as an individual session in which the topics for the sessions were brainstormed, and group sessions were patient-directed.

Nil modifications to the intervention were made, apart from the tailoring of content to each group’s needs.

Intervention adherence and fidelity was assessed by the group facilitator (KOJ) who kept a researcher journal throughout the intervention to record reflections and logistics. A three armed, randomized study comparing the effectiveness of the patient-directed intervention, to a structured intervention and a wait-list control group was planned.

The recruitment target was not met resulting in an amendment of the planned study to a single armed feasibility study with no randomization.

1. Diabetes Queensland. Fact Sheets, 2014. (Available from: http://www.diabetesqld.org.au/health-professionals/resources/fact-sheets.aspx, accessed 2 August 2014).

2. Australian Government Department of Health. Eat for Health: Healthy Eating for Adults. National Health and Medical Research Council (NHMRC);

2014. (Available from: https://www.eatforhealth.gov.au/guidelines: accessed 2 August 2014).

3. Lorig K. Patient education: a practical approach: Sage Publications; USA; 2001. 